\title{
Transplantation of a donor liver with haemochromatosis: evidence against an inherited intrahepatic defect
}

\author{
P C Adams, C N Ghent, D R Grant, J V Frei, W J Wall
}

\begin{abstract}
An iron loaded liver from a 40 year old man with occult haemochromatosis was transplanted into a 19 year old woman with acute liver failure secondary to a paracetamol overdose. Increased parenchymal hepatic iron was found in a liver specimen at biopsy undertaken because of mild rejection $\mathbf{3 0}$ days after transplantation. After transplantation the patient had two episodes of liver rejection confirmed by biopsy. The hepatic iron concentration fell from $161 \mu \mathrm{mol} / \mathrm{g}$ on day 30 after transplant to $26.5 \mu \mathrm{mol} / \mathrm{g}$ (normal $<40$ ) on day 210 . Iron absorption, measured 45 days after transplant, was in the normal range at $12 \cdot 4 \%$. The rapid fall in hepatic iron and the normal iron absorption study result suggest that the genetic defect of haemochromatosis is not exclusively an intrahepatic defect.
\end{abstract}

Departments of

Medicine, Surgery, and Pathology, University Hospital, University of Western Ontario, Canada PC Adams

CN Ghent

D R Grant

JV Frei

W J Wall

Correspondence to:

Dr PC Adams, Department of

Medicine, University

Hospital, PO Box 5339

London, Ontario, Canada N6A 5A5.

Accepted for publication 29 October 1990
Haemochromatosis is a genetic disease of unknown cause that affects approximately one in 400 of the white population.' The disease is characterised by increased intestinal absorption of iron that eventually leads to parenchymal iron deposition in the liver, pancreas, and heart. Organ damage and clinical symptoms do not usually appear until the fifth decade in men and the sixth decade in women. ${ }^{2}$ Theories on the pathogenesis of haemochromatosis have postulated that the primary metabolic defect is in the intestinal mucosa, ${ }^{3-5}$ the reticuloendothelial system, ${ }^{6}$ or the liver. ${ }^{78}$ In this report, we describe the indirect evidence against an intrahepatic defect as the primary defect in haemochromato-

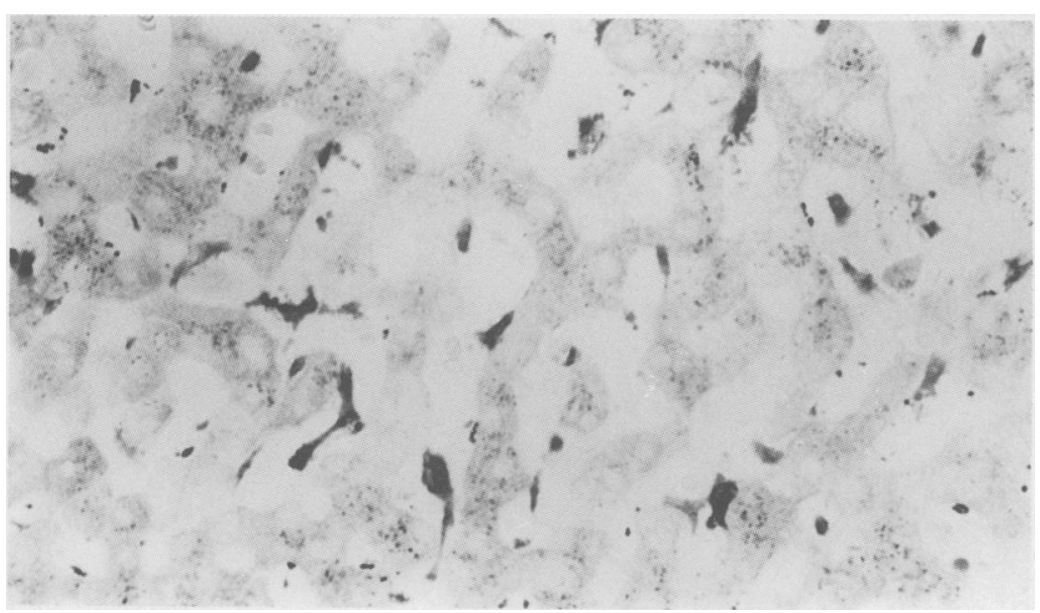

Figure 1: Liver biopsy specimen taken from recipient of iron loaded liver 30 days after transplantation. Iron stain shows increased iron within hepatocytes. Iron staining in Kupffer cells presumably represents iron redistribution in the setting of acute transplant rejection. (Original magnification $\times 200$.) sis provided by inadvertent transplantation of a liver from a patient with haemochromatosis.

\section{Patients and methods}

The donor was a 40 year old Irish man who had a spontaneous cerebral haemorrhage. He had a past history of mild arthritis affecting his hands and shoulders, unexplained dyspnoea on exertion, and a two year history of impotence. There was no history of anaemia, transfusions, or alcohol abuse. Serum, tested posthumously, showed a raised serum ferritin concentration of $1436 \mu \mathrm{g} / \mathrm{l}$ (normal range=15-300). The heart was not used for transplantation because it looked hypokinetic. A necropsy was not performed.

The recipient was a 19 year old woman who developed acute hepatic failure after ingesting approximately $15 \mathrm{~g}$ of paracetamol. She underwent emergency orthotopic liver transplantation receiving $4 \mathrm{U}$ of packed red blood cells. The patient made an excellent postoperative recovery. A percutaneous liver biopsy was performed on day 30 after transplantation because of an increase in aspartate aminotransferase and alanine aminotransferase activities. The biopsy specimen confirmed a diagnosis of acute rejection. An iron stain (Perls' stain) showed 4+ iron in the hepatocytes, ${ }^{9}$ with minimal iron in the Kupffer cells and no fibrosis or cirrhosis (Fig 1). Hepatic iron determination by atomic absorption spectrophotometry ${ }^{10}$ showed a considerable increase in hepatic iron at $161 \mu \mathrm{mol} / \mathrm{g}$ (normal range $0-40 \mu \mathrm{mol} / \mathrm{g}$ dry weight). The estimated hepatic iron index (hepatic iron-age) in the donor was 4.0 which is highly suggestive of genetic haemochromatosis. ${ }^{10}$ Rejection was readily controlled with intravenous methylprednisolone therapy. The serum ferritin value was $2940 \mu \mathrm{g} / \mathrm{l}$ on day 30 and had fallen to $243 \mu \mathrm{g} / \mathrm{l}$ by day 570 . An iron absorption study was performed on day 45 using an oral dose of $1 \mu \mathrm{Ci}{ }^{59} \mathrm{Fe}$ citrate containing $10 \mu \mathrm{mol}$ of ferrous ascorbate. The retained dose of ${ }^{59} \mathrm{Fe}$ at 14 days was calculated with a total body counter. ${ }^{12}$ At the time of the iron absorption study the patient had a haemoglobin concentration of $115 \mathrm{~g} / \mathrm{l}$ and a transferrin saturation value of $23 \%$. The iron absorption was $12 \cdot 4 \%$, which is within normal limits. However, iron absorption is not a diagnostic test for haemochromatosis and the wide range of values for normal volunteers and haemochromatosis patients has been previously described. ${ }^{12}{ }^{13}$ The iron absorption study result in this patient is much less than would be expected for a haemochromatosis patient of this age. ${ }^{13}$ The patient was readmitted to hospital on day 65 and 


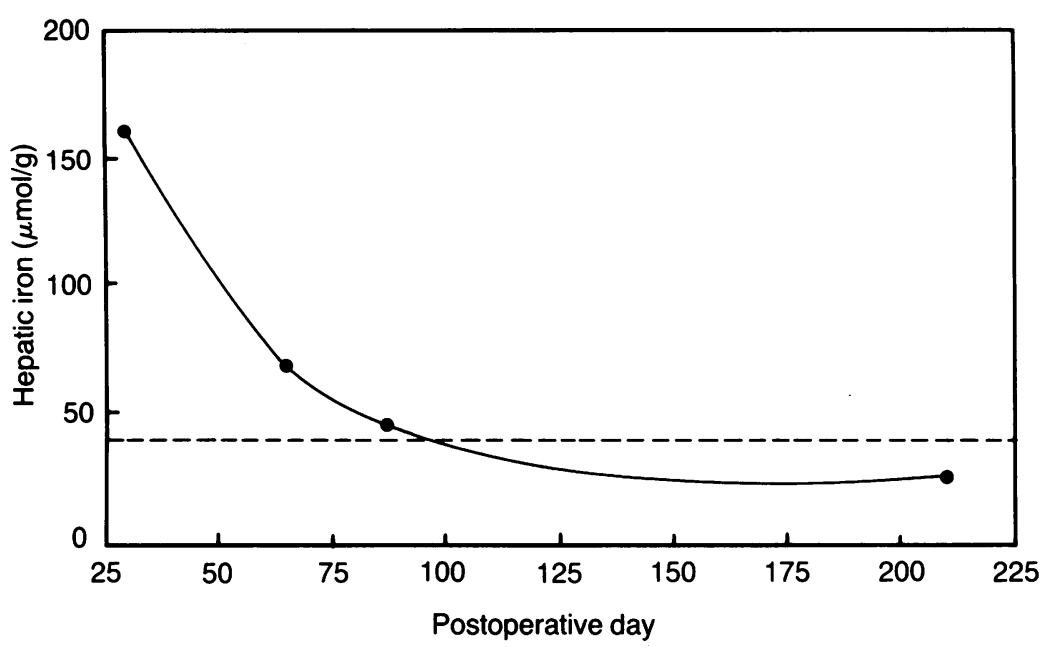

Figure 2: Hepatic iron concentration of transplanted liver after transplantation (normal range $0-40 \mu \mathrm{mol} / \mathrm{g}$ dry weight).
Orthotopic liver transplantation in human beings has provided the opportunity to study the effects of liver replacement on many inherited metabolic diseases. If the metabolic defect of haemochromatosis had been transplanted into this patient, hepatic iron would continue to accumulate as a result of the increased intestinal iron absorption associated with the disease. The rapid reduction of hepatic iron after liver transplantation with normal iron absorption in this patient suggests that the primary metabolic defect of haemochromatosis does not reside exclusively in the liver. However, inflammation in the liver associated with rejection would result in the release of iron into the circulation, redistribution of iron from the parenchyma to macrophages and may contribute to the increased serum ferritin value. Furthermore, the effects of liver transplantation on iron absorption and mobilisation of iron are not clearly understood. Experimental liver transplantation of iron loaded livers in rats has shown some reduction in hepatic iron with rejection but not to the degree seen in this patient. ${ }^{7}$ Experimental iron loading of animals has been shown to cause lipid peroxidation with the production of free radicals that can cause tissue damage. ${ }^{14}$ It is possible that in this patient hepatic rejection led to the release of free iron which further exacerbated the hepatic inflammation. The definitive proof that this patient will not develop progressive iron overload will require long term follow up with assessment of body iron stores by hepatic iron and serum ferritin determination.

\section{Discussion}

This case documents the unusual occurrence of the transplantation of an iron loaded haemochromatosis liver. Considering the prevalence of haemochromatosis of approximately one in 400 , however, ${ }^{1}$ it was inevitable that such a liver would eventually be transplanted inadvertently. Current organ donor procurement and preservation arrangements preclude the routine use of pretransplant liver biopsy assessments and this donor was in the precirrhotic stage of his disease. Since liver function is clinically well preserved until the fifth or sixth decade in haemochromatosis, ${ }^{2}$ it is not surprising that the organ functioned well after transplantation.

The pathogenesis of haemochromatosis remains unknown. Patients with this disorder have increased intestinal absorption of iron which accumulates in the liver, pancreas, and heart causing parenchymal damage over many years. The factors that control iron absorption are poorly understood even in the normal human. A defect in the intestinal regulation of iron absorption has been postulated as the primary defect in haemochromatosis, ${ }^{3-5}$ but iron absorption does respond to body iron stores by decreasing as these increase. ${ }^{13} \mathrm{~A}$ generalised defect in the reticuloendothelial system has also been proposed, with the rapid release of iron by macrophages. ${ }^{6}$ Since haemochromatosis has major clinical sequelae in the liver, an intrahepatic lesion is another possibility. ${ }^{78}$ Increased hepatic iron uptake has been found in patients with haemochromatosis by scanning the liver after the infusion of radiolabelled transferrin. ${ }^{8}$
Dr P C Adams and Dr D R Grant are Career Scientists of the Ministry of Health of Ontario. Dr Adams acknowledges the support of the Medical Research Council of Canada and the secretarial assistance of Cathy Brown.

1 Adams PC, Halliday JW, Powell LW. Early diagnosis and screening for hemochromatosis. Adv Intern Med 1989; 34:

2 Nichols GM, Bacon BR. Hereditary hemochromatosis: pathogenesis and clinical features of a common disease. $A m \mathcal{F}$ Gastroenterol 1989; 84: 851-62.

3 Cox TM, Peter TJ. In vivo studies of duodenal iron uptake in patients with primary and secondary iron storage disease. QF Med 1980; 195: 249-57.

4 Lombard M, Bomford AB, Polson RJ, et al. Differential expression of transferrin receptor in duodenal mucosa in iron overload. Evidence for a site-specific defect in genetic hemochromatosis. Gastroenterology 1990; 98: 976-84.

5 Whittaker P, Skikne BS, Covell AM, et al. Duodenal iron proteins in idiopathic hemochromatosis. $\mathcal{F}$ Clin Invest 1989; 83: $261-7$.

6 Fillet G, Beguin Y, Baldelli L, Model of reticuloendothelial iron metabolism in humans: abnormal behaviour in idioiron metabolism in humans: abnormal behaviour in idio-
pathic hemochromatosis and in inflammation. Blood 1989; pathic hemoch

7 Adams PC, Reece AS, Powell LW, et al. Hepatic iron stores in the control of iron absorption in a rat liver transplantation model. Transplantation 1989; 48: 19-21.

8 Batey RG, Pettit JE, Nicholas AW, et al. Hepatic iron clearance from serum in treated hemochromatosis. Gastroenterology 1978; 75: 856-9.

9 Scheuer PJ, Williams R, Muir AR. Hepatic pathology in relatives of patients with hemochromatosis. $\mathcal{F}$ Patho Bacterol 1962; 84: 53-64.

10 Bassett M, Halliday JW, Powell LW. Value of hepatic iron measurements in early hemochromatosis and determination of the critical iron level associated with fibrosis. Hepatology of the critical irat
$1986 ; 6: 24-9$.

11 Adams PC. Hepatic iron in hemochromatosis. Dig Dis Sci 1990; 35: 690-2.

12 Borwein ST, Ghent CN, Flanagan PR, et al. Genetic and phenotypic expression of hemochromatosis in Canadians. Clin Invest Med 1983; 6: 171-9.

13 Adams PC. Hepatic iron and iron absorption in hemochromatosis. Clin Invest Med 1990; 13: 256-9.

14 Bacon BR, Britton RS. The pathology of hepatic iron overload: a free-radical mediated process? Hepatology 1990; 11 : 127-37. 